\title{
Library of flux-calibrated echelle spectra of southern late-type dwarfs with different activity levels ${ }^{\star}$
}

\author{
C. Cincunegui ${ }^{\star \star}$ and P. J. D. Mauas ${ }^{\star \star \star}$ \\ Instituto de Astronomía y Física del Espacio, CC. 67, suc. 28, 1428 Buenos Aires, Argentina \\ Visiting Astronomers, Complejo Astronómico El Leoncito operated under agreement between the Consejo Nacional de \\ Investigaciones Científicas y Técnicas de la República Argentina and the National Universities of La Plata, Córdoba and \\ San Juan
}

Received 24 April 2003 / Accepted 7 October 2003

\begin{abstract}
We present Echelle spectra of 91 late-type dwarfs, of spectral types from $\mathrm{F}$ to $\mathrm{M}$ and of different levels of chromospheric activity, obtained with the $2.15 \mathrm{~m}$ telescope of the CASLEO Observatory located in the Argentinean Andes. Our observations range from 3890 to $6690 \AA$ at a spectral resolution from 0.141 to $0.249 \AA$ per pixel $(R=\lambda / \delta \lambda \approx 26400)$. The observations were flux calibrated with the aid of long slit spectra. A version of the calibrated spectra is available via the World Wide $\mathrm{Web}^{\dagger}$.
\end{abstract}

Key words. atlases - stars: late-type

\section{Introduction}

Spectral libraries of late-type stars are available for different purposes, and therefore with differing properties. For example, for spectral synthesis of the stellar population of galaxies, a homogeneous and complete library with large spectral range is needed, but the spectral resolution is of only secondary concern. One of the libraries with better spectral resolution used for that purpose is the one by Serote Roos et al. (1996), with only a $1.25 \AA$ A resolution.

This resolution, however, is much lower than needed for studies of chromospheric activity. In fact, stellar activity is seen only in the cores of the strongest transitions, which usually fillin, and only in the most extreme cases go into emission.

Only a few high and mid-resolution libraries of spectra of late-type stars are available. The most relevant for activity

Send offprint requests to: C. Cincunegui,

e-mail: carolina@iafe.uba.ar

* Table 2 is also available in electronic form at the CDS via anonymous ftp to cdsarc.u-strasbg.fr $(130.79 .128 .5)$ or via http://cdsweb.u-strasbg.fr/cgi-bin/qcat?J/A+A/414/699

$\star \star$ Fellow of the CONICET. e-mail: carolina@iafe.uba.ar

$\star \star \star$ Member of the Carrera del Investigador Científico, CONICET. e-mail:pablo@iafe.uba.ar

$\dagger$ The spectra are available as FITS and ascii-files at the URL: http://www.iafe.uba.ar/cincunegui/spectra/Table2 .html. They are also available in electronic form at the CDS via anonymous ftp to cdsarc.u-strasbg.fr $(130.79 .128 .5)$ or via http://cdsweb.u-strasbg.fr/cgi-bin/qcat?]/A+A/414/699. When converting the fits to ascii, the spectra were oversampled to a constant $\delta \lambda \approx 0.15 \AA$. studies are the ones by Montes et al. $(1997,1999)$ and Montes \& Martin (1998). However, these libraries were published to be used for spectral subtraction, and are therefore limited to the least active stars.

On the other hand, the large database by Prugniel \& Soubiran (2001) does not include the spectral region of the $\mathrm{Ca}$ II $\mathrm{H}$ and $\mathrm{K}$ lines, fundamental for studies of stellar activity. Furthermore, this library does not include $\mathrm{dM}$ stars, which are interesting for this kind of work, since the dMe stars show the largest chromospheric line emission observed. Moreover, very large flares are observed in several of these stars, and therefore spectra of their quiescent state can be very useful for stellar flare studies.

Furthermore, these libraries are not flux calibrated (in the last one only the low dispersion spectra are calibrated), which limits its utility for several studies like, for example, to investigate the correlation between different flux indices. Finally, all these libraries are composed of northern stars, due to the large number of two-meter-class telescopes in the northern hemisphere. To our knowledge, no library of spectra of southern dwarfs is available to date.

In this paper we present a library of high resolution echelle spectra of southern late-type dwarfs, covering the range from F6 to M5, and of different levels of activity. In particular, we include some flare stars with very large Ca II H and $\mathrm{K}$ fluxes. These observations were performed as part of a long term project to study variability of stars.

The spectra covers the wavelength range between the $\mathrm{Ca}$ II $\mathrm{H}$ and $\mathrm{K}$ lines on the blue side, and $\mathrm{H}_{\alpha}$ on the red one, which are the most widely used indicators of stellar activity. 
Table 1. Observed standard stars.

\begin{aligned} & \hline \hline HR $\begin{array}{l}\text { Spectral } \\ \text { type }\end{array} \\ &$\hline 718 B9 III \\ & 1996 O9 V \\ & 3454 B3 V \\ & 4468 B9.5 V \\ & 4963 A1 IV \\ & 5501 B9.5 V \\ & 7596 A0 III \\ & 8634 B8 V \\ & 9087 B7 III \\ & \hline\end{aligned}

In the near future, we plan to incorporate the segment covering the Ca II IRT lines and the He $10830 \AA$ line.

\section{The data}

\subsection{Observations}

Our observations were made at the $2.15 \mathrm{~m}$ telescope of the Complejo Astronómico El Leoncito (CASLEO), which is located at $2552 \mathrm{~m}$ above sea level, in San Juan, Argentina. The library is composed of high-resolution echelle spectra obtained with a REOSC spectrograph designed to work between 3500 and $7500 \AA$ and a $1024 \times 1024$ pixel TEK CCD as detector. The maximum wavelength range of our observations is from 3860 to $6690 \AA$ in 24 echelle orders. The spectral resolution ranges from 0.141 to $0.249 \AA$ per pixel $(R=\lambda / \delta \lambda \approx 26400)$.

Since we do not flux-calibrate the echelle spectra directly, we chose a relatively narrow slit (between 200 and $300 \mu$ ) to improve the spectral resolution, despite the loss of some flux.

Due to the strong blaze function of the echelle, it was very difficult to flux calibrate the high-resolution spectra directly. Therefore, for each program star we obtained a mediumresolution long-slit spectrum which we used for the calibration as explained in Sect. 2.3.

The long-slit spectra were obtained with the same spectrograph, replacing the echelle grid by a mirror. The spectral resolution is $\approx 3.4 \AA$ per pixel $(R \approx 1050-2070$ in the observed range $3500-7000 \AA$ ). In this configuration we broadened the slit to $400 \mu$ to assure photometric conditions.

The observations presented here were obtained in three observing runs in 2002. The first was between March 29 and April 1st, the second between August 11 and 14, and the last one between November 21 and 24. In all cases, the first three nights were devoted to the echelle observations, and the last one to the long slit ones. More spectra will be added in the future, as they are obtained and processed.

\subsection{Data reduction: The long-slit spectra}

The long-slit spectra were reduced following the standard procedure. We checked the accuracy of the flat-field images, and decided not to use them because of the noise they introduced.
Besides the program stars, we observed 9 spectrophotometric standards, listed in Table 1, which were processed following the same procedure as the program stars. To check the photometric conditions, we flux-calibrated each standard star with all the others, and obtained in all cases fluxes within $10 \%$ of the ones listed in the $\operatorname{IRAF}^{1}$ database. We then flux calibrated each long-slit program spectra with the closest standard star observed, using the standard IRAF routines.

\subsection{Data reduction: The echelle spectra}

For each star in the sample we took two echelle spectra. On the one hand, this helped us to remove cosmic rays. On the other hand, one should keep in mind that some of these stars show very frequent flares. Since this is the case particularly for the weaker dMe stars, for which integration times as large as two hours are needed, there is a definite possibility of observing a flare and therefore overestimating the activity of the star. The echelle spectra of both images were optimally extracted and wavelength calibrated using IRAF. We visually inspected the Ca II line region of both spectra, and did not find any strong variability. Then we combined both spectra removing cosmic rays.

The blaze function superimposed in each spectroscopic order was too pronounced to normalize the spectra. We flux calibrated each order of the echelle spectra separately according to the following procedure.

First, we reduced the resolution of the flux-calibrated longslit spectra to $13.69 \AA$ (corresponding to 4 pixels), in order to increase the signal-to-noise ratio. These re-sampled program spectra were then used to obtain a sensitivity function with the usual IRAF routines for each order of each program star. We used these sensitivity functions to calibrate each order.

When we calculated this sensitivity function, we took special care with the strongest spectral lines, which are of particular interest for activity studies. Since a small difference in the sensitivity function can strongly affect the strength of the lines, in many cases we had to eliminate several pixels from the fit of the sensitivity function.

Finally, we combined the flux-calibrated echelle orders to obtain a one-dimensional spectrum. We eliminated the extremes of the orders where the signal-to-noise ratio was poor and averaged the remaining overlapping regions of subsequent orders.

As a final step, we integrated the flux to obtain a $V$ magnitude and, when needed, rescaled our observations to the $V$ listed in Table 2 below.

In Fig. 1 the flux-calibrated long-slit and the echelle spectra are shown for HD 158614, over the whole spectral range. To appreciate the good agreement between both spectra, in Fig. 2 we present on a detailed wavelength scale the echelle spectrum re-sampled to $6.85 \AA$, which corresponds to 2 pixels of the the long-slit spectrum, which is also shown.

1 The Image Reduction and Analysis Facility (IRAF) is distributed by the Association of Universities for Research in Astronomy, Inc., under contract to the National Science Foundation. 


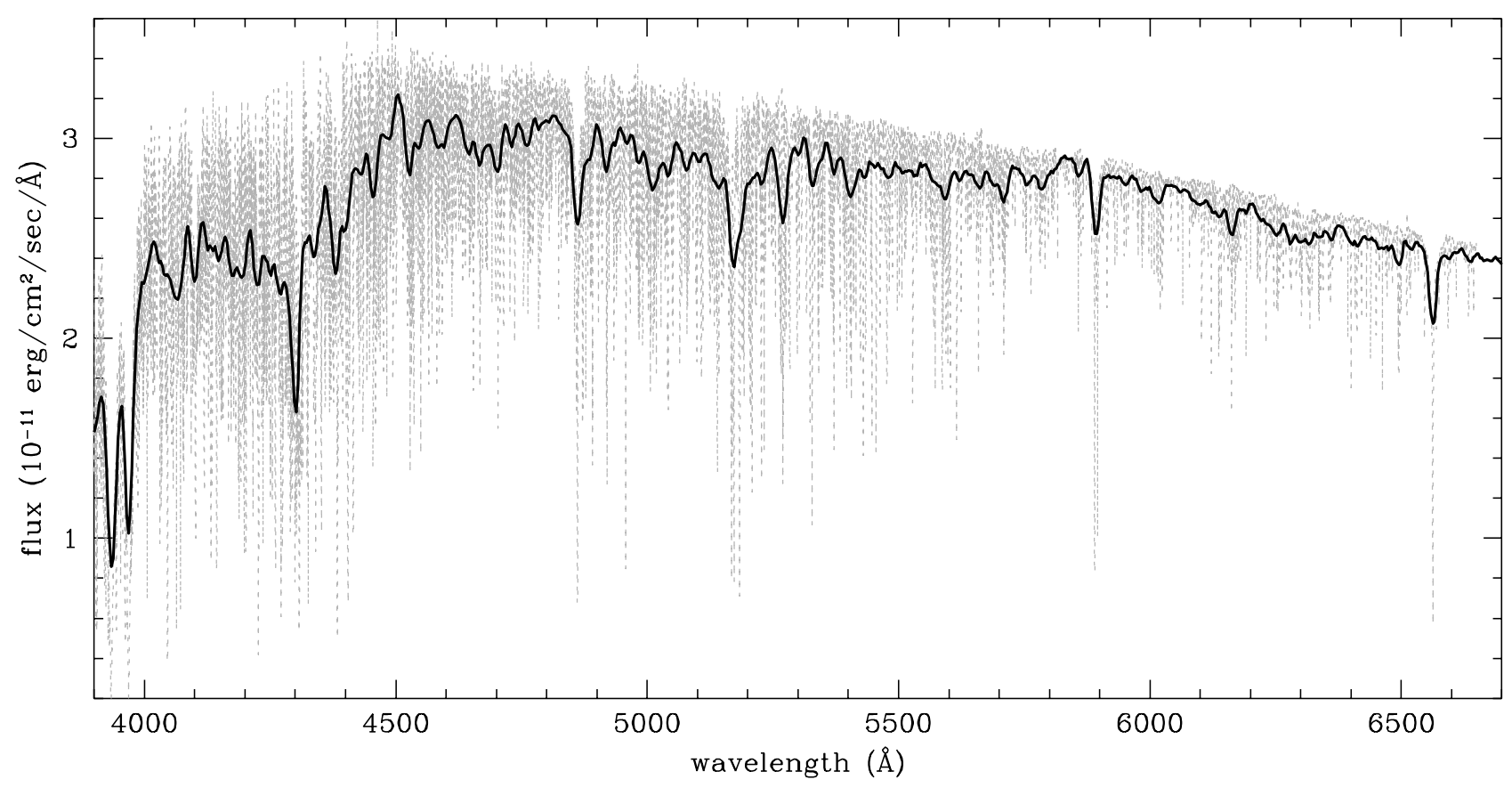

Fig. 1. The low resolution spectrum (solid line), superimposed on the high resolution spectrum (dotted line), for HD 158614. The whole wavelength range of the spectra are shown.

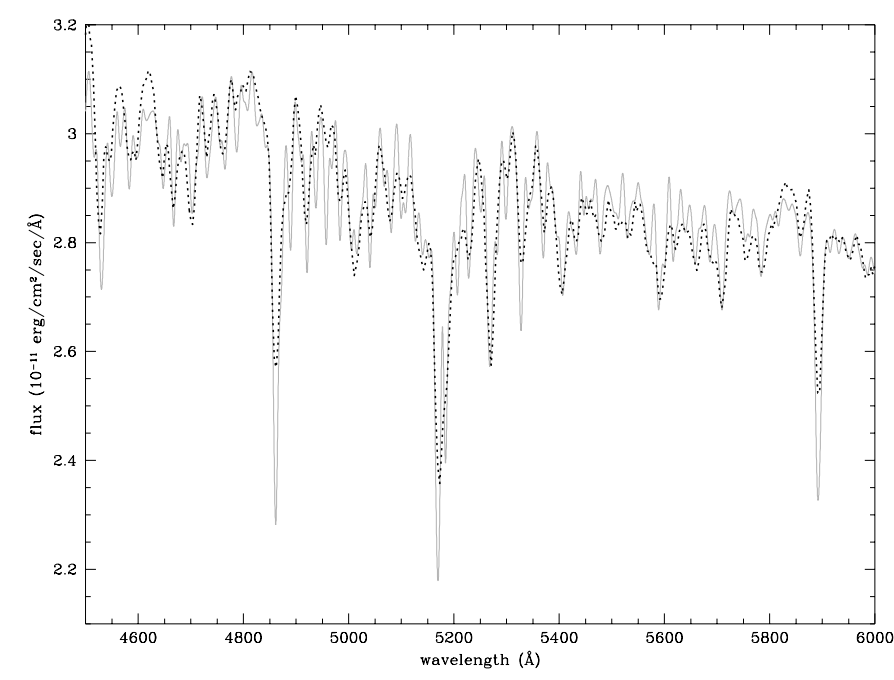

Fig. 2. The high resolution spectrum re-sampled to $6.85 \AA$ (full line) and the low resolution spectrum (dotted line). The star is the same that in Fig. 1.

\section{The library}

In Table 2 we list the stars included in the library. For each star we give the HD and GJ numbers and the star name in the three leftmost columns. In the fourth column we specify which stars are expected to be very active, either because they are dMe stars (with the Balmer lines in emission), or because they belong to the RS CVn or the BY Dra types. In that column we also specify which stars were chosen as non-variable by Duncan et al. (1991), based on the long term database of Mount Wilson observations of the Ca II lines. Also indicated are the stars for which planetary systems have been reported.
In the fifth to tenth columns we list the star's spectral type, its $V$ magnitude, $B-V$ and $U-B$ colors, metallicity (from Cayrel de Strobel et al. 1997, 2001) and trigonometric parallax, from "The Hipparcos and Tycho Catalogue" (ESA 1997), except the ones indicated.

For these active stars, it is important to assess both the activity level and the variability. With this in mind, we include in the table the most widely used index of activity, the $S$ index used at Mount Wilson Observatory, which is defined as the ratio between the flux in the center of the $\mathrm{Ca}$ II $\mathrm{H}$ and $\mathrm{K}$ lines to the continuum nearby. For those stars that have been observed at Mount Wilson, we give in Cols. 11 and 12 both the minimum and maximum values of $S$ that have been observed over the years (from Duncan et al. 1991). For those southern stars that were observed at Cerro Tololo by Henry et al. (1996), we list their value in Col. 13. We also list in Col. 14 the value obtained from our spectra, after the calibration explained in Cincunegui \& Mauas (2002) is performed, using the stars indicated with $\S$ in the table. Finally, we also include the date in which the observation was performed.

Several of our spectra for different spectral type stars are shown in Fig. 3. The low and high resolution flux-calibrated spectra are available via the World Wide Web. Also available through the web is Table 2.

\section{Discussion}

18 of our stars were also observed by Prugniel \& Soubiran (2001). Although in most cases their spectra and ours agree very well, we noted some differences for a few stars. For example, in Fig. 4 we compare their spectrum for HD 9562 with ours (the two lowermost spectra in each panel). To check whether the difference was due to an error in our calibration, we 
Table 2. Observed stars.

\begin{tabular}{|c|c|c|c|c|c|c|c|c|c|c|c|c|c|c|}
\hline HD & GJ & Name & & $\begin{array}{l}\text { Spectral } \\
\text { type }\end{array}$ & $V$ & $B-V$ & $U-B$ & {$[\mathrm{Fe} / \mathrm{H}]$} & Parall. & $\begin{array}{l}S_{\mathrm{MW}} \\
\min \end{array}$ & $\begin{array}{l}S_{\mathrm{MW}} \\
\max \end{array}$ & $S_{\text {Стіо }}$ & $S_{\mathrm{CM}}$ & Date \\
\hline 1835 & 17.3 & BE Cet & $\S \ddagger$ & G3 V & 6.38 & 0.67 & 0.22 & -0.01 & 49.05 & 0.283 & 0.439 & 0.344 & 0.35 & 09.01 .02 \\
\hline 3405 & $24 \mathrm{~A}$ & & & G0/1 V & 6.78 & 0.64 & & & 22.79 & & & & 0.38 & 11.23 .02 \\
\hline 3443 & 25 & & $\S$ & K1 V & 5.57 & 0.71 & 0.20 & $-0.16^{a}$ & 64.38 & 0.149 & 0.782 & 0.185 & 0.18 & 11.23 .02 \\
\hline 3795 & 27.2 & & $\S$ & $\mathrm{G} 3 / 5 \mathrm{~V}$ & 6.14 & 0.70 & 0.21 & -0.70 & 35.02 & 0.137 & 0.171 & 0.145 & 0.16 & 11.23 .02 \\
\hline 4308 & 31.5 & & & G5 V & 6.54 & 0.65 & 0.12 & -0.47 & 45.76 & & & 0.152 & 0.17 & 11.21 .02 \\
\hline 9562 & 59.2 & & $\S$ & G2 IV & 5.76 & 0.60 & 0.24 & 0.18 & 33.71 & 0.113 & 0.155 & 0.146 & 0.14 & 11.23 .02 \\
\hline 10700 & 71 & tau Cet & $\S$ & G8 V & 3.50 & 0.72 & 0.22 & -0.59 & 274.18 & 0.161 & 0.185 & 0.171 & 0.18 & 11.21 .02 \\
\hline 11131 & 9061B & chi Cet B & $\S$ & G0 & 6.72 & 0.60 & 0.12 & -0.06 & 43.47 & & & 0.310 & 0.36 & 11.21 .02 \\
\hline 13445 & 86 & & $\star$ & $\mathrm{K} 1 \mathrm{~V}$ & 6.17 & 0.77 & & -0.21 & 91.63 & & & 0.251 & 0.31 & 11.21 .02 \\
\hline 16673 & 3175 & & $\S$ & F6 V & 5.80 & 0.47 & 0.03 & -0.01 & 46.42 & 0.173 & 0.243 & 0.229 & 0.21 & 09.01 .02 \\
\hline 17051 & 108 & iot Hor & $\star$ & G0 V & 5.40 & 0.57 & & -0.04 & 58.00 & & & 0.225 & 0.23 & 11.23 .02 \\
\hline 17576 & & & & G0 V & 7.92 & 0.52 & & & 6.13 & & & 1.611 & 0.92 & 11.23 .02 \\
\hline 17925 & 117 & EP Eri & $\S \dagger$ & $\mathrm{K} 1 \mathrm{~V}$ & 6.00 & 0.91 & 0.55 & 0.10 & 96.33 & 0.523 & 0.907 & 0.662 & 0.70 & 08.30 .02 \\
\hline 19034 & 121.2 & & & G5 & 8.09 & 0.67 & 0.11 & & 28.33 & & & & 0.17 & 08.30 .02 \\
\hline 19467 & 3200 & & & G3 V & 7.00 & 0.65 & 0.15 & & 31.76 & & & & 0.16 & 08.31 .02 \\
\hline 19994 & 128 & 94 Cet & $\star$ & F8 V & 5.06 & 0.57 & 0.12 & 0.15 & 44.69 & & & & 0.17 & 11.21 .02 \\
\hline 20619 & 135 & & & $\mathrm{G} 1.5 \mathrm{~V}$ & 7.10 & 0.59 & 0.09 & -0.20 & 40.52 & & & & 0.20 & 08.30 .02 \\
\hline 20766 & 136 & zet01 Ret & & $\mathrm{G} 2.5 \mathrm{~V}$ & 5.54 & 0.64 & 0.08 & -0.22 & 82.51 & & & 0.245 & 0.25 & 09.01 .02 \\
\hline 22049 & 144 & eps Eri & $\S \ddagger$ & $\mathrm{K} 2 \mathrm{~V}$ & 3.73 & 0.88 & 0.58 & -0.14 & 310.74 & 0.419 & 0.642 & 0.483 & 0.44 & 08.31 .02 \\
\hline 23249 & 150 & del Eri & $\S \ddagger$ & K0 IV & 3.51 & 0.92 & 0.68 & 0.05 & 110.58 & 0.127 & 0.175 & 0.129 & 0.13 & 08.31 .02 \\
\hline 26965 & $166 \mathrm{~A}$ & DY Eri & $\S$ & K1 V & 4.41 & 0.82 & 0.45 & -0.25 & 198.25 & 0.176 & 0.252 & 0.185 & 0.17 & 09.01 .02 \\
\hline 27442 & 167.3 & eps Ret & $\star$ & K2 IVa & 4.44 & 1.08 & 1.07 & 0.22 & 54.84 & & & & 0.11 & 11.23 .02 \\
\hline 28246 & & & & F6 V & 6.39 & 0.40 & & & 27.00 & & & & 0.20 & 11.21 .02 \\
\hline 30495 & 177 & 58 Eri & $\S$ & G3 V & 5.50 & 0.64 & 0.13 & -0.13 & 75.10 & 0.239 & 0.363 & 0.286 & 0.34 & 09.01 .02 \\
\hline 32147 & 183 & & & K3 V & 6.22 & 1.06 & 0.99 & 0.34 & 113.46 & 0.205 & 0.399 & & 0.28 & 11.21 .02 \\
\hline 35850 & & & & F7 V & 6.31 & 0.50 & & 0.00 & 37.26 & & & & 0.53 & 11.23 .02 \\
\hline 36395 & 205 & & & M 1.5 & 7.92 & 1.52 & 1.21 & $0.60^{a}$ & 175.72 & & & & 2.15 & 09.01 .02 \\
\hline 37572 & & UY Pic & & K0 V & 7.93 & 0.83 & & & & & & 0.952 & 0.74 & 08.31 .02 \\
\hline 38392 & $216 \mathrm{~B}$ & gam Lep B & $\S$ & $\mathrm{K} 2 \mathrm{~V}$ & 6.15 & 0.94 & 0.74 & 0.02 & & & & 0.534 & 0.41 & 09.01 .02 \\
\hline 38393 & $216 \mathrm{~A}$ & gam Lep A & $\S$ & F7 V & 3.60 & 0.47 & & -0.12 & 111.49 & & & 0.182 & 0.17 & 11.22 .02 \\
\hline 38858 & 1085 & & & G4 V & 5.97 & 0.64 & 0.07 & & 64.25 & & & & 0.18 & 11.23 .02 \\
\hline 39917 & & SZ Pic & $\dagger$ & G8 V & 7.90 & 0.76 & & & 5.13 & & & 0.975 & 0.62 & 09.01 .02 \\
\hline 41824 & & & $\dagger$ & G6 V & 6.57 & 0.72 & 0.34 & & 33.64 & & & 0.612 & 0.62 & 09.01 .02 \\
\hline 42581 & $229 \mathrm{~A}$ & & $\triangleleft$ & $\mathrm{M} 1 / 2 \mathrm{~V}$ & 8.14 & 1.51 & & & 173.19 & & & & 1.56 & 09.01 .02 \\
\hline 43587 & $231.1 \mathrm{~A}$ & & & F9 V & 5.71 & 0.61 & 0.08 & -0.08 & 51.76 & 0.131 & 0.173 & & 0.16 & 11.23 .02 \\
\hline 45067 & & & $\S$ & F8 V & 5.90 & 0.53 & 0.05 & -0.16 & 30.22 & 0.115 & 0.167 & 0.148 & 0.16 & 08.31 .02 \\
\hline 45270 & & & & G1 V & 6.53 & 0.56 & & & 42.56 & & & 0.402 & 0.42 & 11.21 .02 \\
\hline 48189 & $3400 \mathrm{~A}$ & & & $\mathrm{G} 1.5 \mathrm{~V}$ & 6.18 & 0.57 & 0.16 & & 46.15 & & & 0.430 & 0.46 & 09.01 .02 \\
\hline 59967 & 3446 & & & G4 V & 6.67 & 0.60 & & & 45.93 & & & 0.384 & 0.41 & 11.22 .02 \\
\hline 75289 & & & $\star$ & G0 I a & 6.36 & 0.58 & 0.10 & 0.28 & 34.55 & & & 0.154 & 0.15 & 03.29 .02 \\
\hline 82106 & 349 & & $\triangleleft$ & K3 V & 7.22 & 1.00 & 0.90 & & 78.87 & 0.797 & 0.797 & & 0.67 & 03.30 .02 \\
\hline 94683 & & & & $\mathrm{~K} 4 \mathrm{~V}$ & 5.97 & 1.78 & 2.01 & & 1.96 & & & & 0.37 & 03.31 .02 \\
\hline 97233 & 416 & & & K4 V & 9.05 & 1.21 & 1.08 & & 44.00 & & & & 0.73 & 03.29 .02 \\
\hline 101581 & 435 & & $\triangleleft$ & $\mathrm{K} 4 / 5 \mathrm{~V}$ & 7.77 & 1.07 & 0.90 & & 79.91 & & & & 0.51 & 03.29 .02 \\
\hline 103112 & $3689 \mathrm{~A}$ & & & K0 & 7.54 & 1.05 & 0.90 & & 12.39 & & & & 0.13 & 03.31 .02 \\
\hline 105115 & & & & $\mathrm{~K} 2 / 3 \mathrm{~V}$ & 6.90 & 1.41 & & & 2.85 & & & & 0.11 & 03.31 .02 \\
\hline 114762 & & & $\star$ & F9 V & 7.30 & 0.55 & -0.07 & -0.82 & 24.65 & & & & 0.17 & 03.29 .02 \\
\hline 117176 & 512.1 & 70 Vir & $\star$ & G4 V & 5.00 & 0.69 & 0.26 & -0.11 & 55.22 & 0.134 & 0.162 & & 0.15 & 03.29 .02 \\
\hline
\end{tabular}


Table 2. continued.

\begin{tabular}{|c|c|c|c|c|c|c|c|c|c|c|c|c|c|c|}
\hline HD & GJ & Name & & $\begin{array}{l}\text { Spectral } \\
\text { type }\end{array}$ & $V$ & $B-V$ & $U-B$ & {$[\mathrm{Fe} / \mathrm{H}]$} & Parall. & $\begin{array}{l}S_{\mathrm{MW}} \\
\mathrm{min}\end{array}$ & $\begin{array}{l}S_{\mathrm{MW}} \\
\max \end{array}$ & $S_{\text {СТIO }}$ & $S_{\mathrm{CM}}$ & Date \\
\hline 118100 & 517 & EQ Vir & $\triangleleft$ & $\mathrm{K} 5 \mathrm{Ve}$ & 9.31 & 1.20 & 1.04 & $-0.15^{a}$ & 50.54 & & & & 3.44 & 03.30 .02 \\
\hline 119850 & 526 & & $\triangleleft$ & M1.5 & 8.46 & 1.45 & 1.09 & & 184.13 & 0.949 & 0.949 & & 1.02 & 03.29 .02 \\
\hline 120136 & $527 \mathrm{~A}$ & tau Boo & $\star$ & F6 IV & 4.50 & 0.48 & & 0.32 & 64.12 & 0.170 & 0.224 & & 0.22 & 03.29 .02 \\
\hline 122303 & 536 & & & M1 & 9.72 & 1.49 & 1.17 & & 98.26 & & & & 0.95 & 03.29 .02 \\
\hline 125072 & 542 & & & K3 V & 6.66 & 1.03 & 0.94 & 0.26 & 84.50 & & & & 0.26 & 03.29.02 \\
\hline 128620 & $559 \mathrm{~A}$ & alf Cen A & & G2 V & -0.01 & 0.71 & 0.22 & 0.22 & 742.24 & & & 0.162 & 0.16 & 08.30.02 \\
\hline 128621 & 559B & alf Cen B & & K1 V & 1.33 & 0.88 & 0.67 & 0.24 & 742.22 & & & 0.209 & 0.23 & 03.29 .02 \\
\hline 130948 & 564 & & & G1 V & 5.88 & 0.55 & 0.00 & -0.20 & 55.73 & & & 0.309 & 0.30 & 03.29 .02 \\
\hline 131977 & $570 \mathrm{~A}$ & & & K4 V & 5.74 & 1.11 & 1.03 & 0.03 & 169.31 & & & 0.709 & 0.43 & 08.31 .02 \\
\hline 144253 & 610 & & & $\mathrm{~K} 3 / 4 \mathrm{~V}$ & 7.40 & 1.04 & 0.94 & -0.03 & 53.93 & & & & 0.21 & 03.31 .02 \\
\hline 146233 & 616 & $18 \mathrm{Sco}$ & & $\mathrm{G} 2 \mathrm{Va}$ & 5.50 & 0.65 & 0.16 & 0.05 & 71.30 & 0.167 & 0.186 & & 0.19 & 03.29 .02 \\
\hline 147513 & $620.1 \mathrm{~A}$ & & & G5 V & 5.38 & 0.60 & 0.14 & 0.03 & 77.69 & & & 0.291 & 0.32 & 09.01.02 \\
\hline 151770 & & & & $\mathrm{G} 3 / 5 \mathrm{~V}$ & 8.34 & 0.65 & & & 7.45 & & & 1.000 & 0.71 & 08.30.02 \\
\hline 152391 & 641 & V2292 Oph & $\S \ddagger$ & G8 V & 6.64 & 0.76 & 0.32 & & 59.04 & 0.278 & 0.482 & 0.431 & 0.40 & 03.29 .02 \\
\hline 156026 & 664 & 36 Oph C & $\dagger$ & K5 V & 6.34 & 1.16 & 0.94 & -0.21 & 167.56 & 0.577 & 1.084 & 1.188 & 0.73 & 08.31 .02 \\
\hline 156425 & & & & K4 V & 7.88 & 1.94 & & -0.20 & -0.32 & & & & 0.31 & 03.31 .02 \\
\hline 157881 & 673 & & & K5 & 7.54 & 1.36 & 1.27 & -0.20 & 129.54 & 1.452 & 1.475 & & 1.76 & 08.30.02 \\
\hline 158614 & 678 & & $\S$ & G9 IV-V & 5.31 & 0.72 & 0.31 & -0.05 & 60.80 & 0.144 & 0.180 & 0.168 & 0.17 & 08.31 .02 \\
\hline 160691 & 691 & mu Ara & & G3 IV-V & 5.15 & 0.70 & 0.23 & 0.20 & 65.46 & & & 0.159 & 0.15 & 03.29 .02 \\
\hline 165185 & 702.1 & & & G5 V & 5.95 & 0.57 & 0.12 & -0.06 & 57.58 & & & 0.333 & 0.33 & 03.29 .02 \\
\hline 172051 & 722 & & & G5 V & 5.87 & 0.63 & 0.17 & & 77.02 & & & 0.181 & 0.19 & 03.30 .02 \\
\hline 173560 & 725.3 & & & $\mathrm{G} 3 / 5 \mathrm{~V}$ & 8.67 & 0.62 & 0.14 & & 11.65 & & & & 0.18 & 03.29.02 \\
\hline 177996 & 4096 & & & K1 V & 7.88 & 0.86 & 0.53 & & 31.48 & & & 0.861 & 0.50 & 03.29 .02 \\
\hline 180617 & $752 \mathrm{~A}$ & & $\triangleleft$ & M2.5 & 9.13 & 1.50 & 1.15 & & 170.25 & 1.050 & 1.453 & & 1.12 & 03.31 .02 \\
\hline 187923 & 4126 & & & G0 V & 6.10 & 0.68 & 0.13 & -0.20 & 36.15 & & & & 0.15 & 03.29 .02 \\
\hline 188088 & 770 & V4200 Sgr & $\ddagger$ & $\mathrm{K} 3 / 4 \mathrm{~V}$ & 6.18 & 1.02 & 0.96 & & 70.34 & & & & 0.59 & 03.30 .02 \\
\hline 189567 & 776 & & & G3 V & 6.07 & 0.64 & 0.07 & -0.30 & 56.45 & & & 0.185 & 0.17 & 03.29 .02 \\
\hline 197076 & 797A & & & G5 V & 6.45 & 0.63 & 0.09 & & 47.65 & 0.158 & 0.180 & & 0.19 & 08.31 .02 \\
\hline 197214 & 4157 & & & G5 V & 6.95 & 0.67 & & & 44.57 & & & 0.188 & 0.32 & 11.22.02 \\
\hline 202628 & 825.2 & & & $\mathrm{G} 2 \mathrm{~V}$ & 6.75 & 0.63 & 0.14 & -0.14 & 42.04 & & & 0.217 & 0.26 & 11.22 .02 \\
\hline 202917 & & & & G5 V & 8.67 & 0.65 & & & 21.81 & & & 0.743 & 0.76 & 11.23 .02 \\
\hline 209100 & 845 & eps Ind & $\triangleleft$ & $\mathrm{K} 4.5 \mathrm{~V}$ & 4.69 & 1.06 & 0.99 & -0.23 & 275.79 & & & 0.668 & 0.40 & 11.22 .02 \\
\hline 210918 & 851.2 & & & G5 V & 6.26 & 0.62 & 0.14 & -0.18 & 45.19 & & & 0.168 & 0.16 & 11.23 .02 \\
\hline 212330 & 857 & & & G3 IV & 5.31 & 0.68 & 0.13 & -0.04 & 48.81 & & & 0.149 & 0.16 & 08.31 .02 \\
\hline 213941 & 863.3 & & & G5 V & 7.59 & 0.66 & 0.12 & -0.42 & 30.98 & & & 0.181 & 0.20 & 11.23 .02 \\
\hline 215768 & & & & G0 V & 7.50 & 0.55 & & & 25.34 & & & 0.318 & 0.33 & 08.31 .02 \\
\hline 219834 & $894.2 \mathrm{~A}$ & 94 Aqr & $\S$ & G6/8 IV & 5.19 & 0.79 & 0.41 & 0.09 & $33.00^{b}$ & 0.139 & 0.181 & 0.145 & 0.16 & 11.23 .02 \\
\hline \multirow[t]{6}{*}{225213} & 1 & & & M1.5 & 8.57 & 1.46 & 0.96 & & 229.32 & & & & 0.33 & 08.31 .02 \\
\hline & 375 & LU Vel & $\triangleleft$ & M3.5 & 11.27 & 1.56 & 0.91 & & 62.88 & & & & 4.68 & 03.30 .02 \\
\hline & 388 & AD Leo & $\triangleleft$ & $\mathrm{M} 3.5 \mathrm{~V}$ & 9.43 & 1.54 & 1.08 & & $213.00^{b}$ & & & & 9.23 & 03.29.02 \\
\hline & 479 & & & M2 & 10.64 & 1.57 & 1.14 & & 103.54 & & & & 2.10 & 03.31 .02 \\
\hline & 551 & Proxima Cen & $\triangleleft$ & M5.5 Ve & 11.05 & 1.97 & 1.43 & & 772.33 & & & & 16.91 & 09.01 .02 \\
\hline & 699 & Barnard's & $\triangleleft$ & M4 Ve & 9.54 & 1.74 & 1.29 & & 549.30 & & & & 0.99 & 09.01.02 \\
\hline
\end{tabular}

Notes: $\star$ : stars with planets.

§: stars reported in CTIO as non-variable, which were used to calibrate the $S$ indexes in Cincunegui \& Mauas (2002).

$\triangleleft$ : dMe stars, or stars presumably active.

$\dagger$ : RS CVn type.

†: BY Dra type.

${ }^{a}$ from Cayrel de Strobel et al. (1997); the rest from Cayrel de Strobel et al. (2001).

${ }^{b}$ from Jenkins (1952); the rest from "The Hipparcos and Tycho Catalogue" (ESA 1997). 


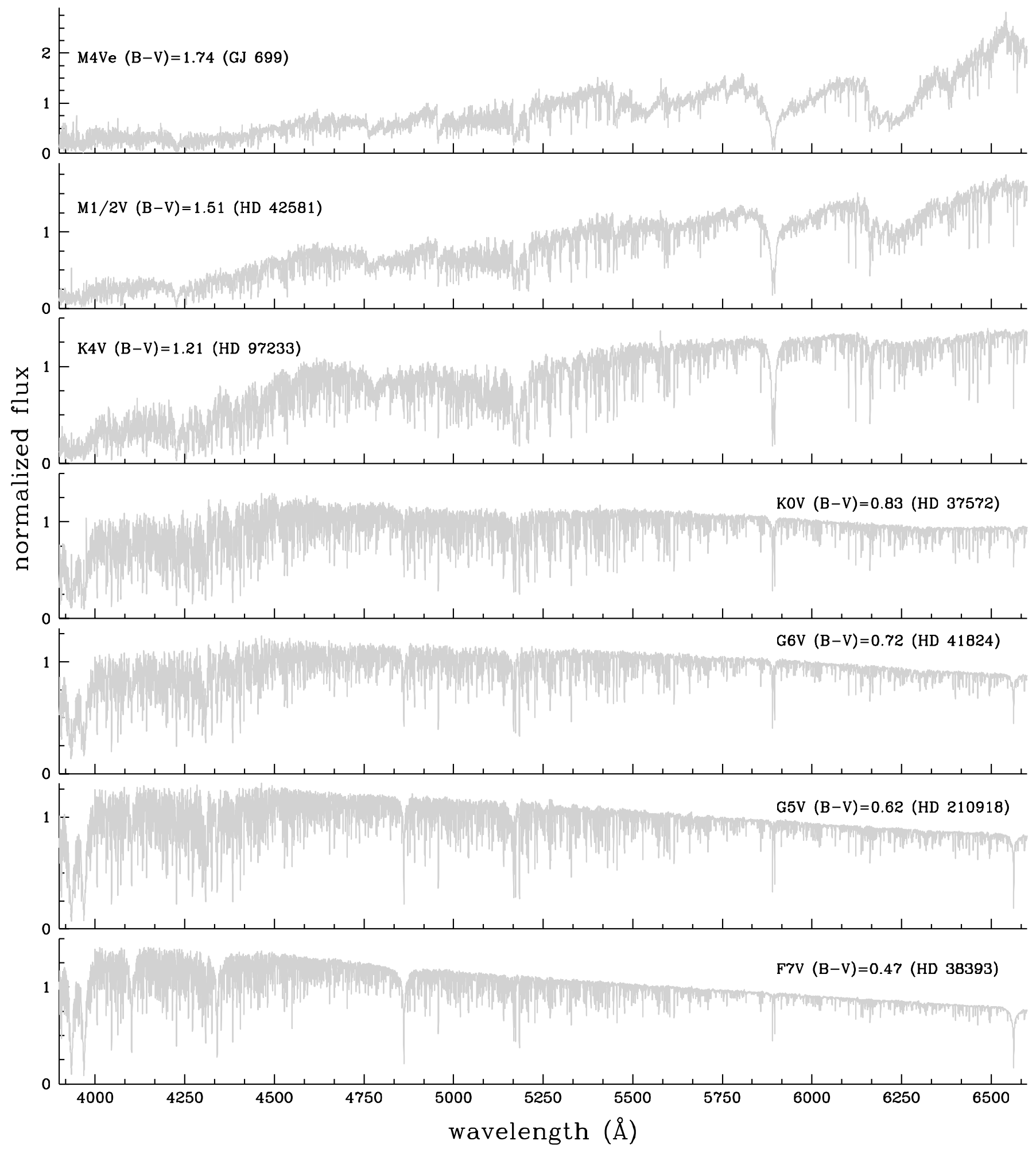

Fig. 3. Some spectra of stars of different types. In each case the spectral type, the colour index and the name of the star is indicated. For a better comparison, the spectra are normalized to flux 1 in the $V$ filter.

compare a lower resolution spectrum for a star of the same spectral type, taken from Silva \& Cornell (1992) with ours resampled to this lower resolution (the two uppermost spectra). We see that these last spectra are practically identical. We have compared all our spectra with both quoted libraries, finding differences smaller than $10 \%$ in most cases.
As an example of the differences in spectra that can be found between stars of the same spectral type but different levels of activity, in Figs. 5 to 7 we compare several of the solartype stars included in our sample, with the Solar flux from the FTS atlas by Brault and Neckel (Neckel 1999), resampled to our resolution. In Fig. 5, the wavelength range is chosen to 

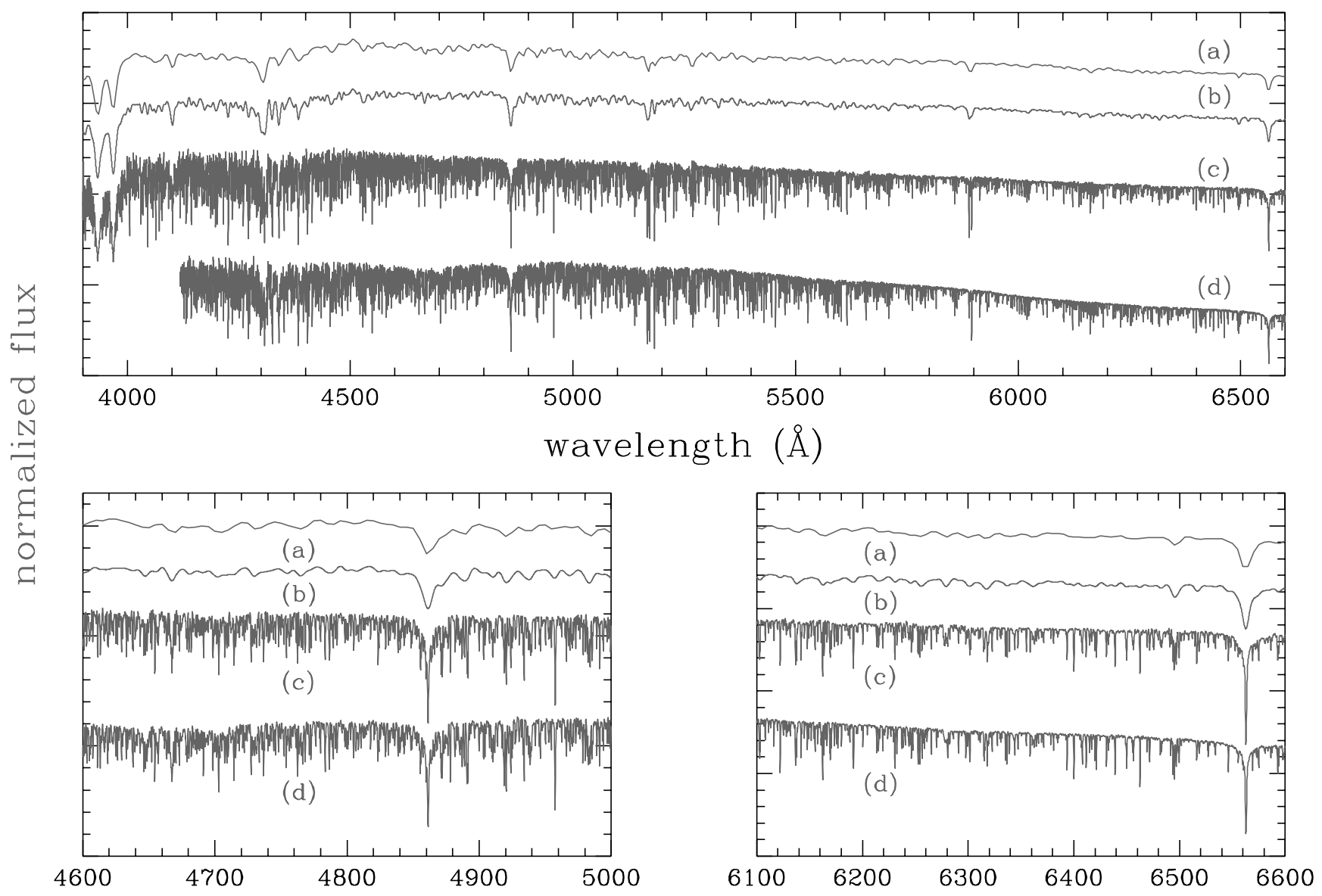

Fig. 4. In the upper panel, we compare our spectrum for HD 9562 (labeled c) with the spectrum of the same star from Prugniel \& Soubiran (2001), resampled to our resolution (labeled d). Also shown, is a spectrum for a star of spectral type G2 IV and $B-V=0.62$ from Silva \& Cornell (1992, labeled a) and ours resampled to the same resolution (labeled b). The flux is normalized as in Fig. 3, and the four spectra are arbitrarily displaced for clarity. In the lower panels, we show details of two spectral regions.

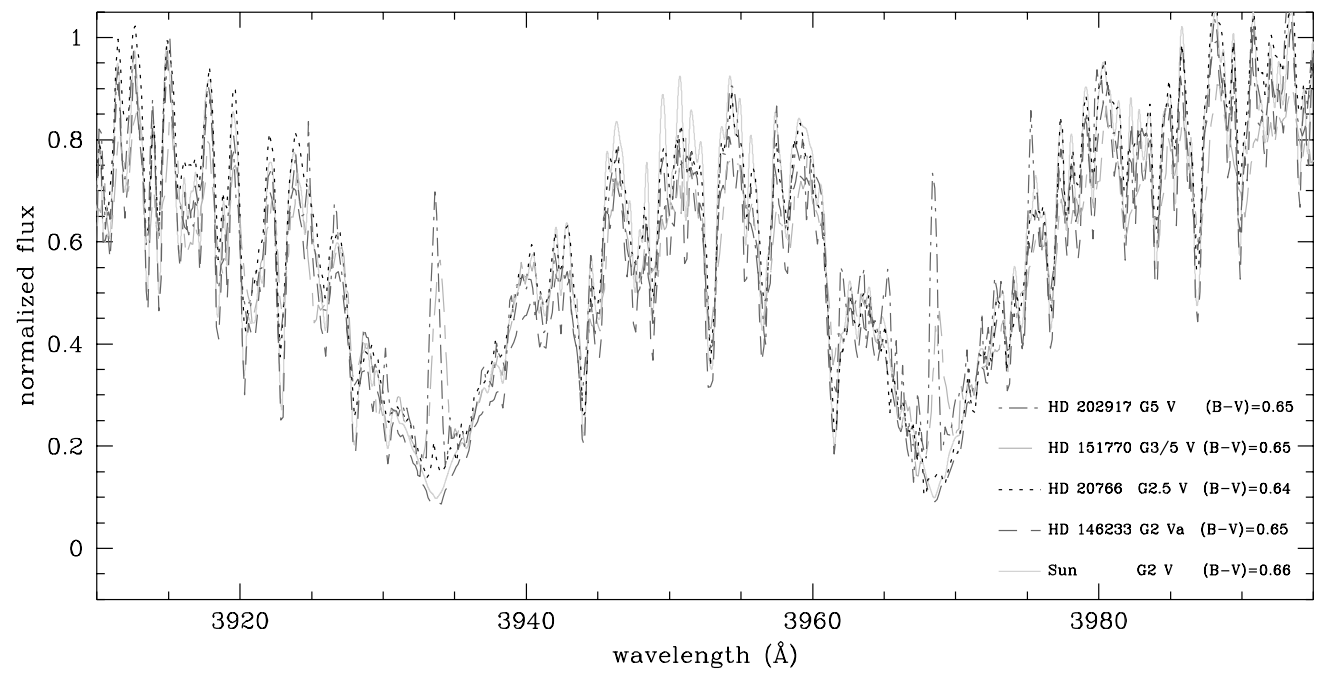

Fig. 5. Several of our solar-type stars and the Sun: the Ca II H and K region. For a better comparison, the spectra are normalized to flux 1 in the $V$ filter.

show the Ca II lines, which are, as previously discussed, the most widely used proxy of chromospheric activity, while in Figs. 6 and 7 we show the Na I $D$ doublet and the $\mathrm{H}_{\alpha}$ line region. As expected, the photospheric contribution for all the stars is practically the same, while the cores of the lines are different, showing that these stars have a very similar photosphere but their levels of activity differ.

Acknowledgements. We would like to thank the director of the CASLEO Observatory, Dr. H. Levato, and all the staff of this institution, for their invaluable help. We also thank Dra. Nidia Morrell for 


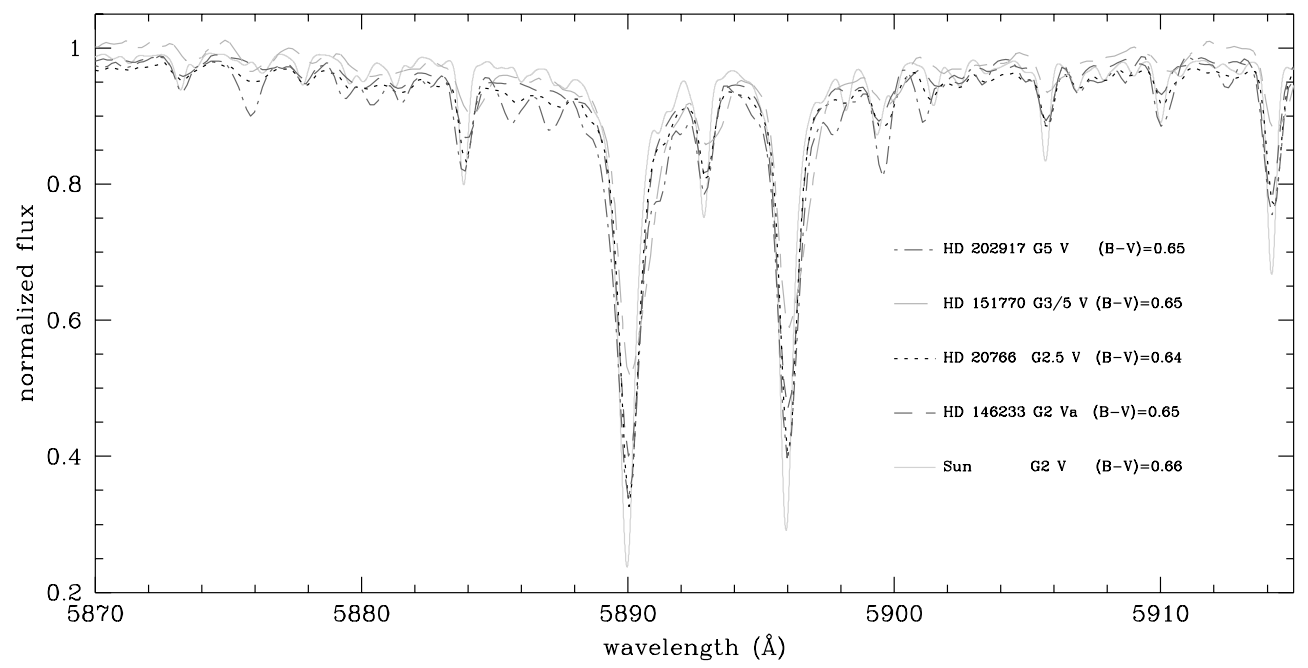

Fig. 6. Several of our solar-type stars and the Sun: the Na I $D$ doublet region. For a better comparison, the spectra are normalized to flux 1 in the $V$ filter.

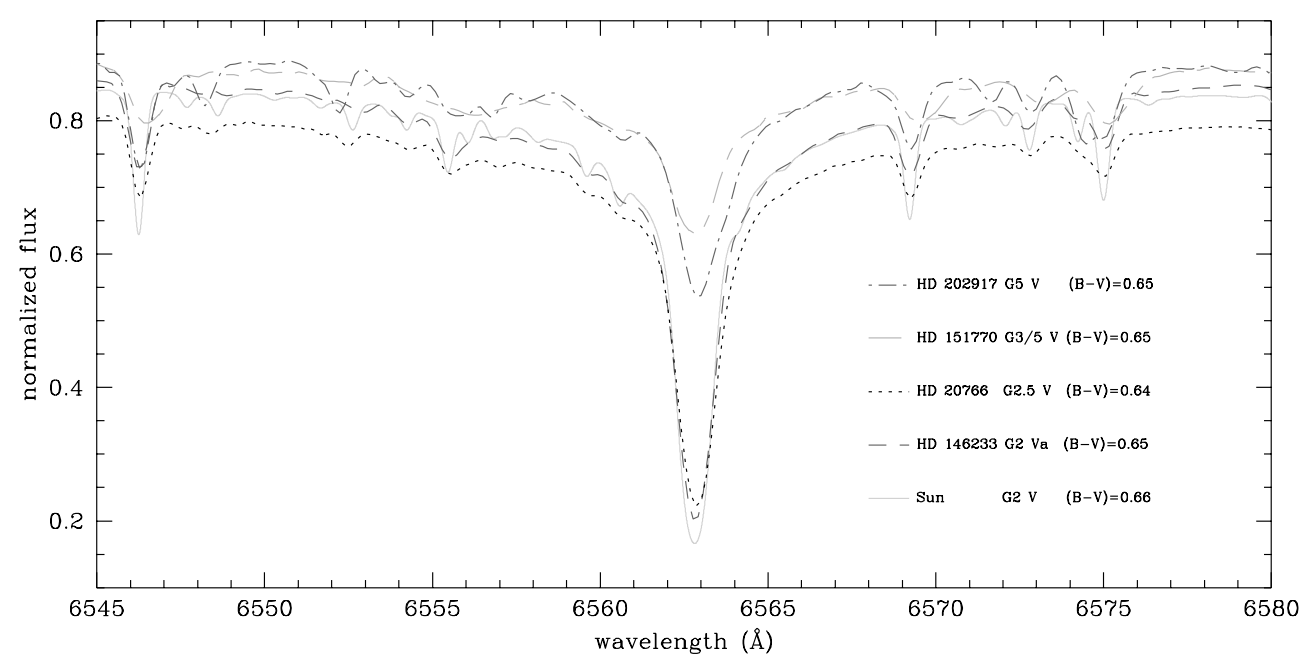

Fig. 7. Several of our solar-type stars and the Sun: the $\mathrm{H}_{\alpha}$ region. For a better comparison, the spectra are normalized to flux 1 in the $V$ filter.

her useful advise, and the unknown referee for many useful comments. The CCD and data acquisition system at CASLEO has been partly financed by R. M. Rich through US. NSF grant AST-90-15827. This work made extensive use of the SIMBAD database, operated at CDS, Strasbourg, France.

\section{References}

Cayrel de Strobel, G., Soubiran, C., Friel, E. D., Ralite, N., \& Francois, P. 1997, A\&A, 124, 299

Cayrel de Strobel, G., Soubiran, C., \& Ralite, N. 2001, A\&A, 373, 159

Cincunegui, C., \& Mauas, P. J. D. 2002, in ESA SP-477, 91

Duncan, D. K., Vaughan, A. H., Wilson, O. C., et al. 1991, ApJS, 76, 383
ESA 1997, The Hipparcos and Tycho Catalogues, ESA SP-1200

Henry, T. J., Soderblom, D. R., Donahue, R. A., \& Baliunas, S. L. 1996, AJ, 111, 439

Jenkins, L. F. 1952, General Catalogue of Trigonometric Stellar Parallaxes, compiled at the Yale Univ. Obs.

Montes, D., \& Martin, E. L. 1998, A\&AS, 128, 485

Montes, D., Martin, E. L., Fernández-Figueroa, M. J., Cornide, M., \& de Castro, E. 1997, A\&AS, 123, 473

Montes, D., Ramsey, L. W., \& Welty, A. D. 1999, ApJS, 123, 283

Neckel, H. 1999, Sol. Phys., 184, 421

Prugniel, P., \& Soubiran, C. 2001, A\&A, 369, 1048

Serote Roos, M., Boisson, C., \& Joly, M. 1996, A\&A, 117, 93

Silva, D. R., \& Cornell, M. E. 1992, ApJS, 81, 865 